\title{
Diabetes Mellitus Predicts Occurrence of Cirrhosis and Hepatocellular Cancer in Alcoholic Liver and Non-alcoholic Fatty Liver Diseases
}

\author{
Evan J. Raff ${ }^{1}$, Donny Kakati ${ }^{1}$, Joseph R. Bloomer ${ }^{2}$, Mohamed Shoreibah ${ }^{2}$, Khalid Rasheed ${ }^{3}$ \\ and Ashwani K. Singal*2 \\ ${ }^{1}$ Department of Internal Medicine, UAB, Birmingham, AL, USA; ${ }^{2}$ Division of Gastroenterology and Hepatology, UAB, Birmingham, \\ $A L$, USA; ${ }^{3}$ Department of Internal Medicine, UAB Montgomery Program, Huntsville, $A L$, USA
}

\begin{abstract}
Background and Aims: Alcohol abuse and nonalcoholic fatty liver disease (NAFLD) are common causes of liver disease. Diabetes mellitus (DM) is a common comorbidity among NAFLD patients. We performed this study with the specific aim to examine the impact of DM on progression of alcoholic liver disease (ALD) liver and NAFLD. Methods: Medical charts of 480 patients with ALD or NAFLD (2004-2011) managed at a tertiary center were retrospectively reviewed. NAFLD was diagnosed based on exclusion of other causes of liver disease and alcohol use of $<10 \mathrm{~g} / \mathrm{d}$. ALD was diagnosed based on alcohol use of $>40 \mathrm{~g} / \mathrm{d}$ in women or $>60 \mathrm{~g} / \mathrm{d}$ in men for $>5$ years. Results: Of 480 patients (307 NAFLD), 200 diabetics differed from nondiabetics for: age ( $52 \pm 11$ vs. $49 \pm 11$ years; $p=0.004)$; male gender (48\% vs. $57 \% ; p=0.03$ ); metabolic syndrome ( $49 \%$ vs. $30 \% ; p=0.0002)$; NAFLD ( $80 \%$ vs. $56 \%$; $p<0.0001)$; cirrhosis (70\% vs. $59 \% ; p=0.005)$; and hepatocellular carcinoma (HCC; $8 \%$ vs. $3 \% ; p=0.009)$. Over a 3 year median follow-up period, diabetics relative to nondiabetics had a higher probability to develop cirrhosis (60\% vs. $41 \%$; $p=0.022)$ and HCC ( $27 \%$ vs. $10 \% ; p=0.045)$. There was a trend for increased development of hepatic encephalopathy in diabetics compared to nondiabetics ( $55 \%$ vs. $39 \% ; p=0.053$ ), and there was no difference between the two groups in survival or other liver disease complications. Conclusions: DM increased risk for cirrhosis and HCC among patients with ALD and NAFLD. Prospective studies with longer follow-up periods are needed to examine the impact of DM on survival and the role of aggressive HCC screening in diabetic cirrhotics.

(C) 2015 The Second Affiliated Hospital of Chongqing Medical University. Published by XIA \& HE Publishing Ltd. All rights reserved.
\end{abstract}

Keywords: Diabetes mellitus; Steatohepatitis; Nonalcoholic steatohepatitis; Alcoholic cirrhosis.

Abbreviations: ALD, alcoholic liver disease; ALT, alanine aminotransferase; AST, aspartate aminotransferase; BMI, body mass index; CCI, charlson comorbidity index; $\mathrm{CI}$, confidence interval; $\mathrm{CT}$, computed tomography; DM, diabetes mellitus; HCC, hepatocellular carcinoma; MELD, model for end-stage liver disease; MRI, magnetic resonance imaging; NAFLD, non-alcoholic fatty liver disease; $\mathrm{NASH}$, nonalcoholic steatohepatitis; OR, odds ratio.

Received: 05 January 2015; Revised: 19 February 2015; Accepted: 22 February 2015

DOI: $10.14218 / \mathrm{JCTH} .2015 .00001$.

*Correspondence to: Ashwani K Singal, University of Alabama at Birmingham, 1808 7th Ave South, BDB 351, Birmingham, AL 35294, USA. Tel: +1-205-975-

3515, Fax: +1-205-975-9777, E-mail: ashwanisingal.com@gmail.com

\section{Introduction}

Alcohol abuse and nonalcoholic fatty liver disease (NAFLD) are the most common causes of liver cirrhosis and indications for liver transplantation in the US, following chronic hepatitis $C$ virus infection. ${ }^{1}$ Although alcoholic liver disease (ALD) and NAFLD exhibit different phenotypes and risk factors, they share similar pathogenic mechanisms and histological findings of steatohepatitis. ${ }^{2}$ The histological spectrum may range from simple steatosis to more advanced disease, including steatohepatitis, fibrosis, cirrhosis, or hepatocellular carcinoma (HCC).

There is an epidemic of obesity in the US, and the frequency of liver disease and liver transplantation due to steatohepatitis in NAFLD (NASH) has been increasing over the last two decades. Prevalence of NASH is currently reported to be as high as $17 \%$ among patients with NAFLD. ${ }^{3-5}$ It is estimated that $8 \%$ of the US population has diabetes mellitus (DM), and it is the seventh leading cause of death in the US. ${ }^{6}$ Furthermore, the prevalence of DM is reported to be higher among patients with liver disease secondary to NAFLD relative to other etiologies of liver disease. . $^{7,8}$

Many studies have reported DM to be a risk factor in patients with ALD and NAFLD for the development of fibrosis, accelerated fibrosis progression, and liver disease related mortality. ${ }^{9-16}$ Multiple cohort and case-control studies have also shown an association between diabetes and HCC. ${ }^{17-23}$ However, data remain relatively scant on the link between diabetes and progression to cirrhosis and development of associated complications. Here, we explored this association in a cohort of patients with ALD and NAFLD.

\section{Material and methods}

\section{Study population}

Patients evaluated at a single tertiary referral center between January 2004 and December 2011 diagnosed with ALD (ICD09 codes 571.0 and 571.3), alcoholic cirrhosis of the liver (571.2), cirrhosis of the liver without alcohol (571.5), or other chronic NAFLD including steatosis (571.8) formed the study population. Patients were excluded from the analysis if details regarding alcohol use were unavailable or if alcohol consumption was $>10 \mathrm{~g} / \mathrm{d}$ in patients diagnosed with NALFD (Fig. 1). 
Evan J.R. et al: Diabetes and steatohepatitis

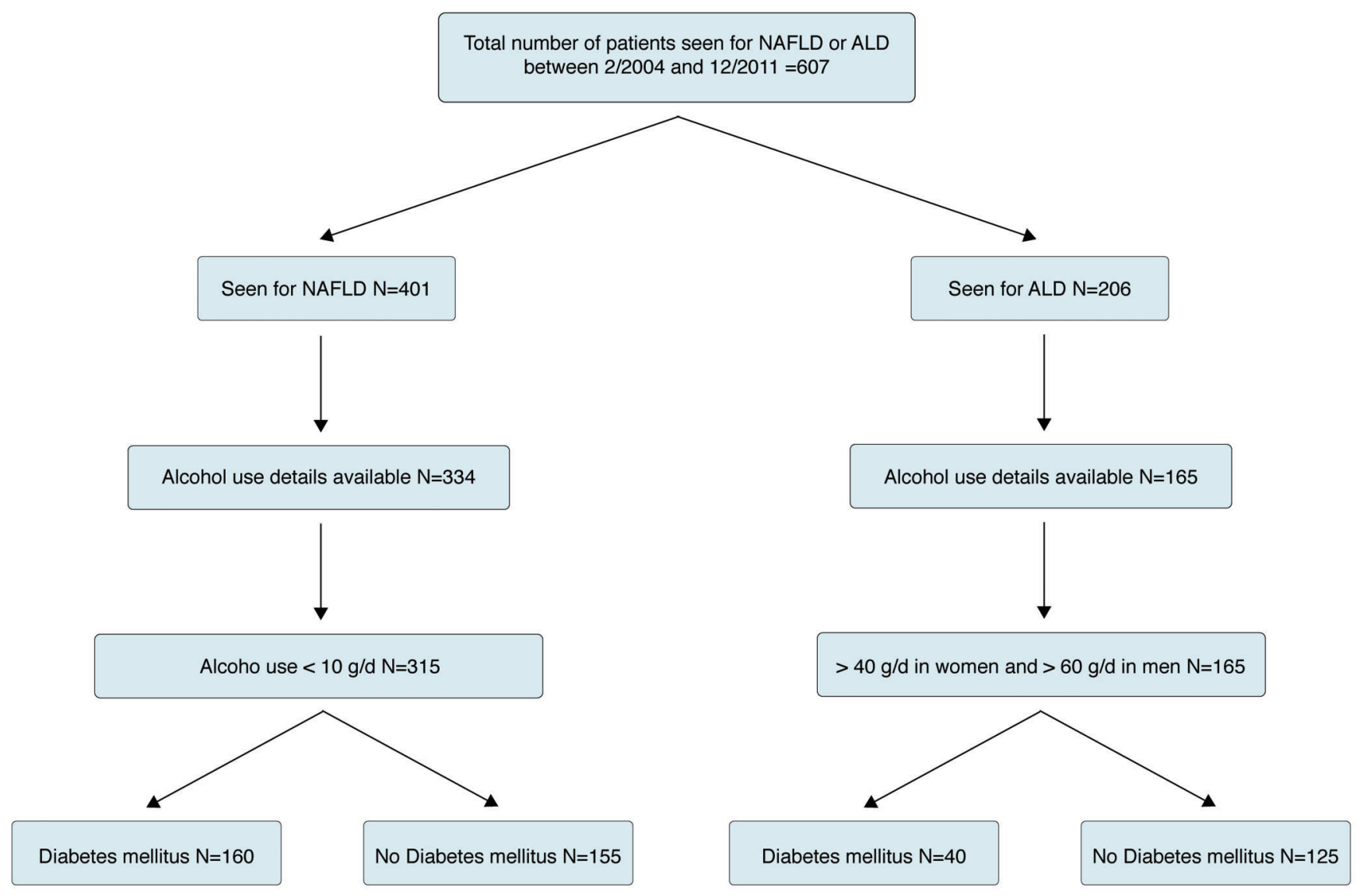

Fig. 1. Study population

\section{Definitions}

ALD

Defined based on the following criteria: 1) presence of liver disease as determined by clinical evaluation along with laboratory work-up, imaging assessment, or liver biopsy findings; 2) exclusion of other causes of liver disease; and 3 ) a history of excessive alcohol use $(>40 \mathrm{~g} / \mathrm{d}$ in women or $>60 \mathrm{~g} / \mathrm{d}$ in men for $>5$ years).

\section{NAFLD}

Defined based on the following criteria: 1 ) demonstration of hepatic steatosis by imaging or biopsy; 2) exclusion of significant alcohol consumption (>10 g/d); and 3) exclusion of other causes of hepatic steatosis. There was considerable variation across different studies for the amount of alcohol consumption used to define NAFLD, and some studies did not take into account gender differences in alcohol use. ${ }^{3}$ Therefore, we used the safest and lowest amount of alcohol use $(<10 \mathrm{~g} / \mathrm{d})$ to define NAFLD in our study cohort.

\section{Steatohepatitis}

This is a histological diagnosis based on the presence of steatosis and inflammation (lobular inflammation and hepatocyte ballooning). NAFLD activity score (NAS) was determined on each biopsy as the underweighted sum of the scores for steatosis: grades $0-3$ as proportion of hepatocytes containing fat vacuoles with $<5 \%, 5-33 \%, 33-66 \%$, and $>66 \%$ hepatocytes, respectively; lobular inflammation: grades $0-3$ as inflammation foci per $200 \times$ field with absent, $<2$ foci, $2-4$ foci, and $>4$ foci, respectively; and cytological ballooning: grades 0-2 qualitatively as number of ballooned cells with none, few, and many cells, respectively. ${ }^{24}$ Due to the lack of a scoring system to grade steatohepatitis in ALD patients and in order to keep homogeneity for diagnosis of steatohepatitis, we used the same definition of NAS, four or more to define steatohepatitis in the ALD group. Diagnosis of steatohepatitis was made on biopsies in ALD and NAFLD patients with an NAS of 3 or more.

\section{Alcohol use}

Grams per day derived from the average number of drinks consumed per day. One drink was equal to 12 ounces of beer, 5 ounces of wine, or 1.25 ounces of hard liquor, each representing $15 \mathrm{~g}$ of pure alcohol.

\section{Diabetes}

Based on the following criteria: 1) formal diagnosis listed in the medical chart; and/or 2 ) receiving specific antidiabetic 
medications; and/or 3) documented hemoglobin A1c of $6.5 \%$ or above. ${ }^{25}$

\section{Metabolic syndrome}

Defined as the presence of three or more of the following: hyperglycemia (taking medications for previously diagnosed type 2 diabetes or fasting blood sugar level $\geq 100 \mathrm{mg} / \mathrm{dL}$ ), hypertension (taking medications for previously diagnosed hypertension or blood pressure $\geq 130 / 85 \mathrm{mmHg}$ ), obesity (body mass index (BMI) $\geq 30$ ), reduced high density lipoprotein level $(<50 \mathrm{mg} / \mathrm{dL}$ in females and $<40 \mathrm{mg} / \mathrm{dL}$ in males), and/or elevated triglyceride level $(\geq 150 \mathrm{mg} / \mathrm{dL}){ }^{26-28}$

Cirrhosis

Diagnosed by clinical/imaging criteria and/or biopsy when available.

HCC

Diagnosed based on American Association for Study of Liver Diseases guidelines and criteria incorporating computed tomography (CT) and magnetic resonance imaging (MRI) scans and/or biopsy when available. ${ }^{29}$

\section{Data collection}

Our center utilized a searchable electronic medical record that collects information on patient demographic, clinic notes, notes from hospitalizations (history and physicals, progress notes, and discharge summaries), vital signs, reports from radiologic imaging studies, laboratory data, and pathology reports. The referral center had utilized this system to store information since 2000.

Using the medical record number as a unique identifier, patient charts were reviewed for data collection on patient demographics (age, gender and race); BMI; dates of onset of symptoms and of diagnosis; components of metabolic syndrome (diabetes, hypertension, dyslipidemia, and obesity); alcohol intake in $\mathrm{g} / \mathrm{d}$; and presentation with cirrhosis and/or associated complications (ascites, variceal bleeding, hepatic encephalopathy, and HCC). Results of laboratory, imaging, upper gastrointestinal endoscopy, and liver biopsy details were also recorded. Charlson comorbidity index (CCI) was obtained for each patient using the information on defined comorbidities. ${ }^{30,31}$ For patients with available liver biopsy information, data were collected for steatosis, lobular inflammation, and hepatocyte ballooning. Data were also collected for fibrosis stage: $0-4$ as no fibrosis, portal fibrosis, periportal fibrosis, bridging fibrosis, and cirrhosis, respectively. ${ }^{32,33}$

Data on prospective follow-up of patients from the time of their first contact at our center was collected retrospectively. Development of cirrhosis was evaluated among patients without diagnosis of cirrhosis at or within the first 6 months of presentation. Development of liver disease complications (ascites, hepatic encephalopathy, variceal hemorrhage, and $\mathrm{HCC}$ ) was assessed among cirrhotics who did not have these complications at or within 1 month of their presentation. Patient survival status was recorded from the chart review and confirmed with the National Death Registry. Time to development of cirrhosis, liver disease complications, and death was calculated from dates of event occurrence and disease onset. Patients lost to follow-up and those without the event at the time of their last follow-up were censored. Medical charts of these patients were reviewed to identify patients meeting criteria for diagnosis of ALD and NAFLD, as previously detailed in the section on definitions.

\section{Statistical analyses}

Patients with and without DM were compared for demographics; components of metabolic syndrome; CCI; presentation with cirrhosis and/or associated complications; laboratory data; and findings on endoscopy, imaging, and liver biopsy. Chi-square and student's tests were utilized for categorical and continuous variables, respectively. Logistic regression models were built to examine predictors of cirrhosis and HCC development. Variables different between the two groups and other clinically relevant ones were entered into the model. Results are reported as odds ratio (OR) with 95\% confidence interval (CI). Kaplan Meier curves were generated to compare diabetics and nondiabetics for development of cirrhosis, complications of liver disease, and patient survival. Log rank test was used for statistical significance. All statistical analyses were performed using the Statistical Analyses Software (SAS Institute, USA), and $p<0.05$ was considered statistically significant. The study was approved by the Institutional Review Board at our center.

\section{Results}

\section{Study population}

Of 607 patients in the database (401 with diagnosed NAFLD), 108 were excluded from analysis (67 NAFLD) because details were missing regarding alcohol use. A total of 19 NAFLD patients were excluded due to reported alcohol use of $>10 \mathrm{~g} / \mathrm{d}$ and did not meet our criteria for NAFLD diagnosis. Of the remaining 480 patients (315 NAFLD), 200 (160 NAFLD) met the criteria for diagnosis of diabetes (Fig. 1).

\section{Baseline characteristics}

Diabetics relative to nondiabetics were older in age, predominantly female in gender, had metabolic syndrome, and had NAFLD as the underlying etiology (Table 1). About $90 \%$ of patients were Caucasian, consistent with the patient population at our center. Diabetics had a higher BMI $(35 \pm 8$ vs. $31 \pm 10 ; p<0.0001)$ and were more likely to have hypertension $(69 \%$ vs. $48 \% ; p<0.0001)$ than nondiabetics. Triglyceride levels were similar and lower density lipoprotein levels were lower among diabetics than nondiabetics $(153 \pm 136$ vs. $139 \pm 111 \mathrm{mg} / \mathrm{dL} ; p=0.3$ and $98 \pm 36$ vs $111 \pm 53 \mathrm{mg} / \mathrm{dL} ; \quad p=0.02$, respectively). Diabetics had a higher CCI, after excluding the impact of liver disease and diabetes, than nondiabetics (Table 1). In total, 305 (64\%) patients had cirrhosis on initial evaluation, and this percentage was higher in diabetics (Table 1 ). A total of 128,40 , and 33 patients had ascites, encephalopathy, and variceal bleeding, respectively, at presentation, and there were no differences between diabetics and nondiabetics. Twenty-four patients had HCC at presentation, with a higher proportion in diabetic patients (Table 1). There was over a 3-fold risk for cirrhosis and/or HCC in diabetics at the time of clinical presentation (Table 2). Other significant predictors of cirrhosis and $\mathrm{HCC}$ were age, male gender, and the ratio of aspartate aminotransferase (AST) to alanine aminotransferase (ALT) 
Evan J.R. et al: Diabetes and steatohepatitis Table 1. Demographic and clinical characteristics of patients with alcoholic and non-alcoholic fatty liver diseases comparing patients with and without diabetes

\begin{tabular}{|c|c|c|c|c|}
\hline Variable & & $\begin{array}{l}\text { No diabetes mellitus } \\
\qquad(n=280)\end{array}$ & Diabetes mellitus $(n=200)$ & $p$-value \\
\hline \multicolumn{5}{|c|}{ Demographics } \\
\hline Age (years) & & $49 \pm 11$ & $52 \pm 11$ & 0.004 \\
\hline Male (\%) & & 57 & 48 & 0.03 \\
\hline Caucasians (\%) & & 89 & 87 & 0.97 \\
\hline \multicolumn{5}{|c|}{ Comorbidities } \\
\hline MS (\%) & & 30 & 49 & 0.0002 \\
\hline $\mathrm{CCI}$ & & $1.6 \pm 2$ & $2.7 \pm 2.2$ & $<0.0001$ \\
\hline \multicolumn{5}{|c|}{ Liver disease status at presentation } \\
\hline NAFLD (\%) & & 56 & 80 & $<0.0001$ \\
\hline Cirrhosis (\%) & & 59 & 70 & 0.005 \\
\hline $\mathrm{HCC}(\%)$ & & 3 & 8 & 0.009 \\
\hline Ascites (\%) & & 30 & 22 & 0.052 \\
\hline PSE (\%) & & 7 & 7 & 0.37 \\
\hline Variceal bleed (\%) & & 9 & 7 & 0.93 \\
\hline \multicolumn{5}{|c|}{ Laboratory values } \\
\hline ALT (IU/L) & & $55 \pm 56$ & $46 \pm 43$ & 0.03 \\
\hline AST (IU/L) & & $67 \pm 68$ & $53 \pm 48$ & 0.004 \\
\hline MELD score & & $11 \pm 8$ & $9 \pm 8$ & 0.03 \\
\hline \multicolumn{5}{|c|}{ Endoscopic findings } \\
\hline \multirow[t]{3}{*}{ EV } & Absent & 35 & 35 & 0.44 \\
\hline & Small & 32 & 27 & \\
\hline & Moderate-to-large & 33 & 38 & \\
\hline \multirow[t]{3}{*}{ PHG } & Absent & 56 & 60 & 0.07 \\
\hline & Mild-to-moderate & 40 & 34 & \\
\hline & Severe & 4 & 6 & \\
\hline
\end{tabular}

ALT, alanine aminotransferase; AST, aspartate aminotransferase; CCI, charlson comorbidity index; EV, esophageal varices; HCC, hepatocellular carcinoma; MELD, Model for End-Stage Liver Disease; MS, metabolic syndrome; NAFLD, nonalcoholic fatty liver disease; PHG, portal hypertensive gastropathy; PSE, portal systemic encephalopathy.

levels (Table 2). Other variables included in the model were race/ethnicity, CCI, and etiology of liver disease (NAFLD or ALD).

Diabetics compared to nondiabetics had lower alanine ALT and aspartate AST levels (Table 1). However, the AST/ALT ratio was similar $(1.4 \pm 1.1$ vs. $1.5 \pm 0.9 ; p=0.23)$ between the two groups. About $15 \%$ of patients were positive for autoimmune markers, and there was no difference between those with and without diabetes ( $16 \%$ vs. $15 \% ; p=0.45)$. Serum albumin levels were similar between the two groups as

Table 2. Predictors of cirrhosis and HCC in alcoholic and non-alcoholic fatty liver diseases

\begin{tabular}{lcccccc}
\hline & \multicolumn{2}{c}{ Predictors of cirrhosis } & $p$ & \multicolumn{2}{c}{ Predictors of HCC } & $p$ \\
\hline Variable & OR & $95 \%$ CI & & OR & $95 \%$ CI & \\
Diabetes & 3.9 & $2.3-6.4$ & $<0.0001$ & 3.0 & $1.3-6.9$ & $<0.0001$ \\
Age increase by 5 years & 1.30 & $1.17-1.44$ & $<0.0001$ & 1.17 & $1.02-1.53$ & 0.047 \\
Male gender & 2.1 & $1.3-3.4$ & 0.002 & 2.8 & $1.3-9.2$ & 0.001 \\
ALD vs. NAFLD & 14.7 & $7.6-28.3$ & $<0.0001$ & 11.2 & $5.2-17.2$ & $<0.0001$ \\
Cirrhosis & & & & 8.9 & $1.4-75$ & $<0.0001$ \\
\hline
\end{tabular}

ALD, alcoholic liver disease; ALT, alanine aminotransferase; AST, aspartate aminotransferase; CI, confidence interval; HCC, hepatocellular carcinoma; NAFLD, nonalcoholic fatty liver disease; OR, odds ratio. 
Evan J.R. et al: Diabetes and steatohepatitis

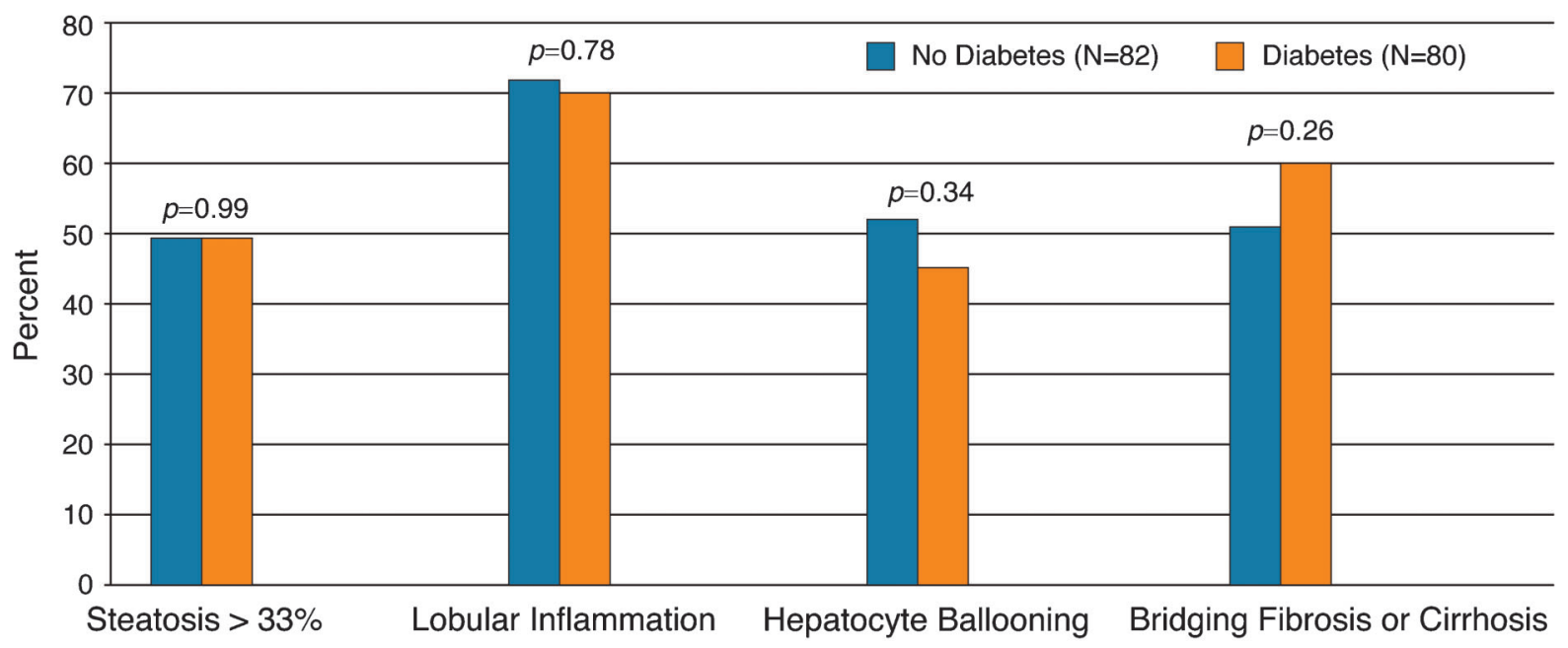

Fig. 2. Histologic findings in liver biopsies among patients with alcoholic liver disease (ALD) or nonalcoholic fatty liver disease (NAFLD) and with or without diabetes.

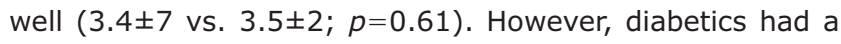
lower model for end-stage liver disease (MELD) score compared to nondiabetics (Table 1). On upper gastrointestinal endoscopy, there were no differences in the presence and severity of portal hypertensive gastropathy or esophageal varices between diabetics and non-diabetics (Table 1).

\section{Histological findings}

Liver biopsy details on histological findings were available in 162 patients ( 80 with DM; Fig. 2). When comparing diabetics and nondiabetics, there were no differences in steatosis in
$>33 \%$ of hepatocytes ( $49 \%$ vs. $49 \% ; p=0.99$ ), lobular inflammation ( $72 \%$ vs. $70 \% ; p=0.78)$, and hepatocyte ballooning ( $52 \%$ vs $45 \% ; p=0.34)$. NAFLD activity score was also similar between the two groups (3.6 1.7 vs. $3.4 \pm 1.5 ; p=0.61$ ). Steatohepatitis (NAFLD activity score of 4 or more) was present in $44(27 \%)$ cases, and 20 of these were diabetic and only two had ALD. About $55 \%$ patients had bridging fibrosis or cirrhosis, and there were no differences between those with or without DM (60\% vs. $51 \% ; p=0.26)$. ALD patients were more likely than NAFLD patients to have cirrhosis at the time of first contact ( $46 \%$ vs. $12 \%$, $p<0.0001)$. Among NAFLD patients with diabetes, there was

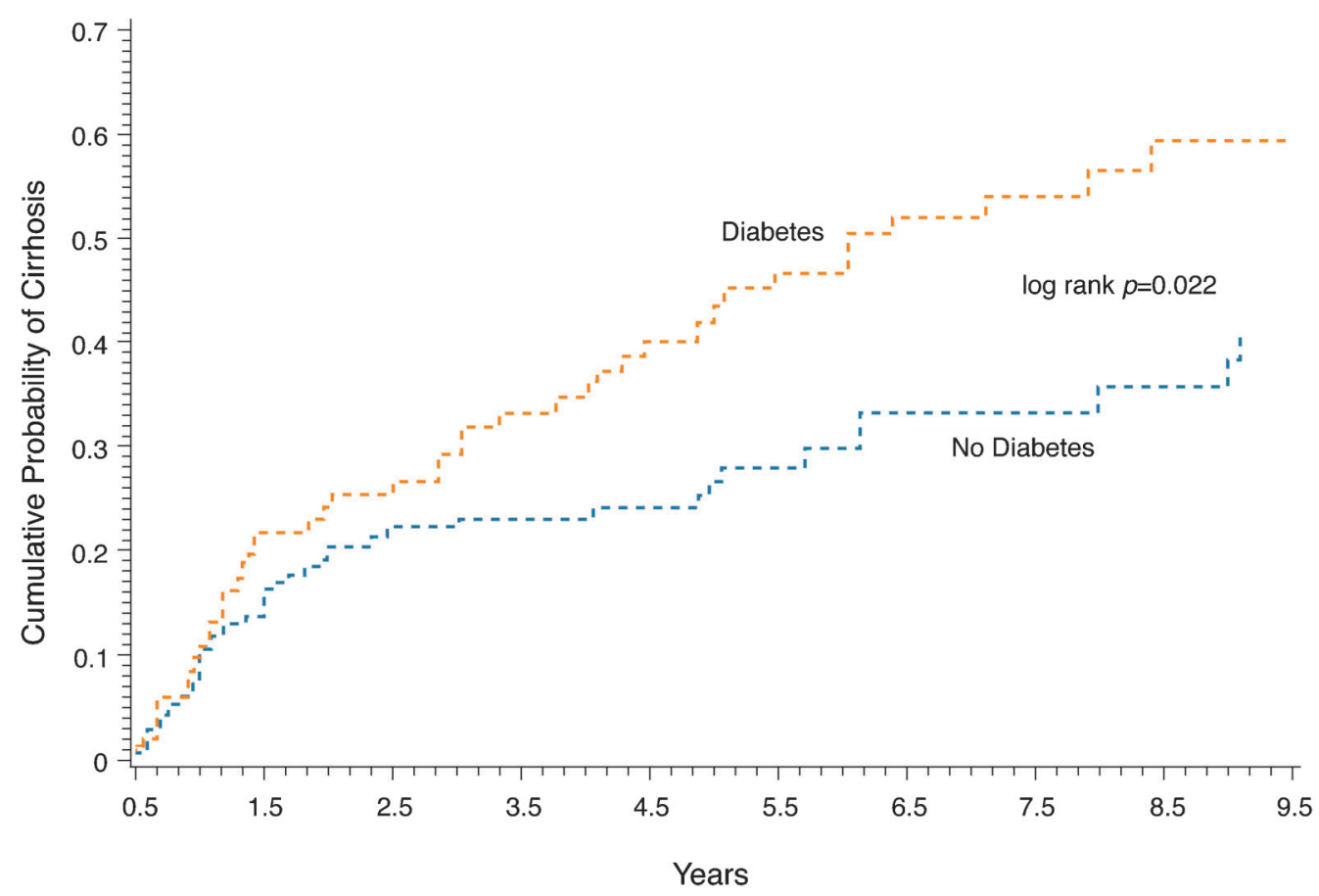

Fig. 3. Cumulative probability of developing cirrhosis in diabetics and nondiabetics in patients with alcoholic liver or non-alcoholic fatty liver diseases. The probability of developing cirrhosis is higher among diabetics. 
Evan J.R. et al: Diabetes and steatohepatitis

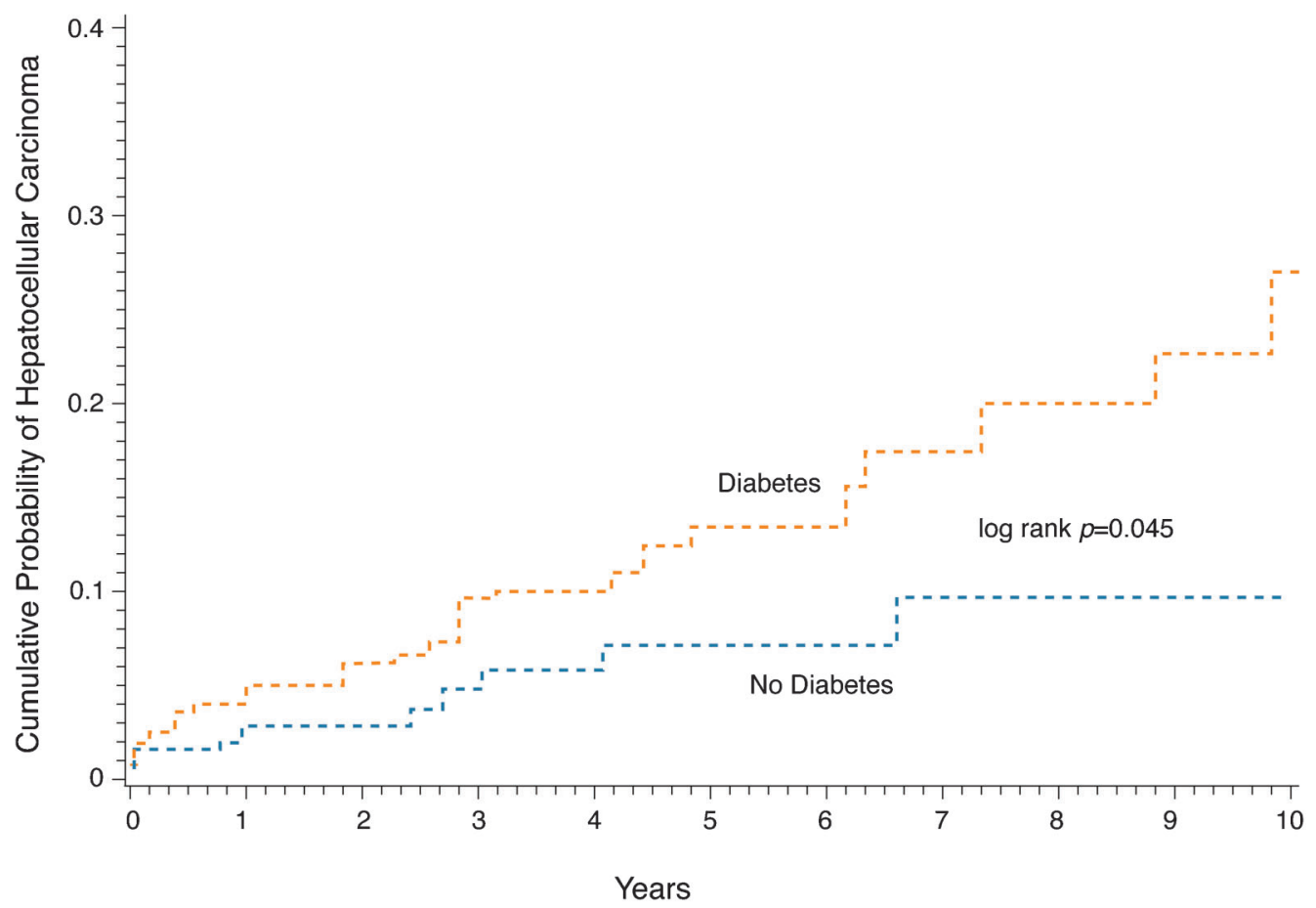

Fig. 4. Cumulative probability of developing hepatocellular carcinoma (HCC) in diabetics and nondiabetics in patients with alcoholic liver or non-alcoholic fatty liver diseases. Results show that the probability of developing HCC is higher among diabetics.

an increased tendency to have cirrhosis, as determined by biopsy, than nondiabetics ( 5 of 56 vs. 1 of $49, p=0.21$ ). The frequency of cirrhosis at first contact based on clinical and/or histological diagnosis of cirrhosis was also higher in diabetics than nondiabetics (51 of 160 vs. 24 of $155, p=0.009$ ).

\section{Impact of diabetes on progression of liver disease}

\section{Development of cirrhosis and HCC}

Over a median follow-up period of 6 months among 156 patients ( 55 with DM) without cirrhosis, a higher proportion of diabetics relative to nondiabetics developed cirrhosis (43\% vs. $27 \%$, respectively), with a higher cumulative probability of developing cirrhosis ( $60 \%$ vs. $41 \%$, respectively; log rank $p=0.022$, Fig. 3). Similarly, over a median follow-up of 3 years among 359 patients with cirrhosis at or during followup, a higher proportion of diabetics compared to nondiabetics developed HCC ( $22 \%$ vs. $5 \%$, respectively) with a higher cumulative probability of developing HCC ( $27 \%$ vs. $10 \%$; log rank $p=0.045$, Fig. 4). Etiology specific analyses were also performed. Among ALD patients, the cumulative development of cirrhosis and HCC for diabetics and nondiabetics was $97 \%$ vs. $87 \%, p=0.023$ and $13 \%$ vs. $6 \%, p=0.08$. Similar figures were found for NAFLD patients, $64 \%$ vs. $36 \%$, $p<0.0001$ and $4.5 \%$ vs. $1.5 \%, p=0.38$ respectively.

\section{Development of liver disease complications}

Over a median follow-up period of 3 years among patients without liver disease complications at or within 30 days of disease onset, diabetics relative to nondiabetics tended to develop more frequently hepatic encephalopathy (30\% vs. $9 \%$, respectively) with a higher cumulative probability (39\% vs. $55 \%$, respectively; log rank $p=0.053$ ). However, probabilities of developing ascites and variceal bleeding were similar between diabetics and nondiabetics (52\% vs. $42 \%$; $p=0.3$ and $23 \%$ vs. $16 \%, p=0.47$, respectively). Etiology specific analyses for cumulative development of hepatic encephalopathy was higher among diabetics with NAFLD than nondiabetics ( $35 \%$ vs. $15 \%, p=0.0006)$. There was a trend suggesting diabetics with ALD were at greater risk than nondiabetics for developing hepatic encephalopathy (35\% vs. $28 \%, p=0.43)$.

\section{Overall survival}

In total, 31 of 480 (6\%) patients in the study population died, and 17 of these were nondiabetics. There was no difference in probability of survival between patients with and without DM over a median follow-up period of 3.2 and 4 years, respectively ( $85 \%$ vs. $83 \%$, respectively; $p=0.81$ ).

\section{Discussion}

Our analysis of patients with steatohepatitis-related liver disease revealed: a) different baseline characteristics and similar histological findings of patients with or without DM; b) diabetes is a risk factor for the development of cirrhosis and $\mathrm{HCC}$; and c) diabetics relative to nondiabetics are more likely to develop cirrhosis and HCC.

Baseline differences between diabetics and nondiabetics were likely due to a higher proportion of NAFLD patients in the diabetic group. Many studies have reported NAFLD patients to 
be older females with a higher likelihood of exhibiting components of metabolic syndrome. ${ }^{4,5,34}$ Histological findings on liver biopsy were similar between diabetics and nondiabetics. Furthermore, the proportion of patients with advanced fibrosis or cirrhosis on liver biopsy was similarly stratified for DM. These results were likely due to selection bias due to the performance of liver biopsy, as the proportion of patients with cirrhosis at first contact and follow-up remained higher among diabetics relative to nondiabetics. ${ }^{35,36}$ In one study of 46 NAFLD patients (17 with type 2 diabetes), markers of insulin resistance correlated independently with the degree of hepatic inflammation and fibrosis. ${ }^{37}$ In another study on NASH patients; older age, obesity and DM predicted severe liver fibrosis on biopsy. A higher proportion of NAFLD patients in the diabetes group than the nondiabetes group and the selection bias for performing liver biopsy may explain the differences between these two patient groups found in our retrospective analysis.

Many studies have shown that type DM is a predictor of fibrosis, accelerated fibrosis progression, and increased liverrelated mortality. ${ }^{9-14}$ Similarly, studies have observed DM to be a risk factor for the development of HCC in patients with cirrhosis. ${ }^{20,38-40}$ Our study findings are consistent with these reports and demonstrated that DM is a predictor for cirrhosis and/or HCC irrespective of the etiology of steatohepatitisrelated liver disease. In one study, the synergistic effects of alcohol abuse and DM on the development of HCC was shown, suggesting that heavy alcohol consumption may exacerbate the effect of DM on the development of cirrhosis and HCC. ${ }^{39}$

The mechanisms underlying DM mediated acceleration of liver disease progression in patients with steatohepatitis remain unclear. It has been speculated that release of free fatty acids from adipose tissue due to insulin resistance in DM accumulate within hepatocytes. Insulin resistance also mediates release of cytokines, such as leptin and tumor necrosis factor-alpha, which in turn mediate activation of inflammatory pathways ${ }^{41}$ and mitochondrial oxidative stress within the hepatocytes. ${ }^{42}$ Furthermore, adiponectin, an anti-inflammatory cytokine produced by adipose tissue, is decreased in states of insulin resistance. ${ }^{43,44}$ Local inflammation and circulating adipokines stimulate stellate cells to produce collagen, connective tissue growth factor, and extracellular matrix, ultimately resulting in fibrosis. ${ }^{45}$ Risk of HCC has been shown to be modulated by antidiabetic medications. Insulin use was associated with increased risk and use of oral drugs, including metformin and thiazolidinediones, was linked with decreased risk. ${ }^{46,47}$ In the current study, the lack of details on diabetic medications limited analysis on the impact antidiabetic medications have on the risk of cirrhosis and HCC.

We found that diabetics with ALD or NAFLD related liver disease had an increased likelihood of developing encephalopathy without an increase in risk for ascites or variceal bleeding. Similar findings were reported in another study on patients with decompensated cirrhosis. In this study, diabetics relative to nondiabetics had a higher prevalence and severity of hepatic encephalopathy. There were no significant differences between the two groups, however, in terms of Child-Pugh class, MELD scores, or the presence of ascites and esophageal varices. ${ }^{48}$ In the future, mechanistic studies should be performed to identify pathways mediating this effect of diabetes on the development of hepatic encephalopathy among patients with cirrhosis. Interestingly, in spite of the increased risk for developing cirrhosis and HCC in diabetics, the overall survival was similar to nondiabetics. In another study that evaluated patients with compensated liver cirrhosis, diabetes was associated with a significant increase in mortality. ${ }^{49}$ This discrepancy with our data may be due to the relatively short follow-up period (median around 3 years) in our study.

The advantages of the current study include its large cohort and the well-characterized study population of steatohepatitis-related liver disease patients. Limitations include its retrospective design, patient population from a single center, and lack of details on alcohol use, control of NAFLD risk factors (including diabetes and weight), and antidiabetic medications. Prospective studies with a larger sample size and longer follow-up period are suggested in order to examine: 1) the role of antidiabetic medications on the development of cirrhosis and its complications in patients with steatohepatitis-related liver disease; 2) the effect of diabetes on clinical end points, including overall survival and transplantation, and 3 ) the benefit of more aggressive HCC screening in diabetics with steatohepatitis-related liver disease.

\section{Conclusions}

Diabetes is a risk factor for the development of cirrhosis and HCC in the natural history of ALD and NAFLD. Our study findings are of clinical relevance and demonstrate the need for a) better management and control of diabetes and b) more rigorous screening and surveillance of HCC among patients with steatohepatitis-related liver disease.

\section{Conflict of interest}

None

\section{Author contributions}

Performing data collection (EJR, DK, MS, KR), conceptualizing and designing the study as well as performing data analysis (AKS), reviewing the manuscript (AKS, JRB).

\section{References}

[1] Singal AK, Anand BS. Recent trends in the epidemiology of alcoholic liver disease. Clin Liver Dis 2013;2:53-56. doi: 10.1002/cld.168.

[2] Völzke H. Multicausality in fatty liver disease: is there a rationale to distinguish between alcoholic and non-alcoholic origin? World Gastroenterol 2012;18:3492-3501. doi: 10.3748/wjg.v18.i27.3492.

[3] Chalasani N, Younossi Z, Lavine JE, Diehl AM, Brunt EM, Cusi K, et al. The diagnosis and management of non-alcoholic fatty liver disease: practice guideline by the American Gastroenterological Association, American Association for the Study of Liver Diseases, and American College of Gastroenterology. Gastroenterology 2012;142:1592-1609. doi: 10.1053 /j.gastro.2012.04.001.

[4] Charlton MR, Burns JM, Pedersen RA, Watt KD, Heimbach JK, Dierkhising RA. Frequency and outcomes of liver transplantation for nonalcoholic steatohepatitis in the United States. Gastroenterology 2011;141:1249-1253. doi: 10.1053/j.gastro.2011.06.061.

[5] Singal AK, Guturu P, Hmoud B, Kuo YF, Salameh H, Wiesner RH. Evolving Frequency and Outcomes of Liver Transplantation Based on Etiology of Liver Disease. Transplantation 2013;95:755-760. doi: 10.1097/TP.0b013 e31827afb3a.

[6] http://www.cdc.gov/diabetes/data/statistics/2014statisticsreport.html, accessed October 24, 2014.

[7] Palmeira CM, Rolo AP, Berthiaume J, Bjork JA, Wallace KB. Hyperglycemia decreases mitochondrial function: The regulatory role of mitochondria biogenesis. Toxicol Appl Pharmacol 2007;225:214-220. doi: 10.1016/j.taap 2007.07.015. 
[8] Williamson RM, Price JF, Glancy S, Perry E, Nee LD, Hayes PC, et al. Prevalence of and risk factors for hepatic steatosis and nonalcoholic Fatty liver disease in people with type 2 diabetes: the Edinburgh Type 2 Diabetes Study. Diabetes Care 2011;34:1139-1144. doi: 10.2337/dc10-2229.

[9] Wanless IR, Lentz JS. Fatty Liver Hepatitis (Steatohepatitis) and Obesity - An Autopsy Study with Analysis of Risk Factors. Hepatology 1990;12:11061110. doi: 10.1002/hep.1840120505.

[10] Younossi ZM, Gramlich T, Matteoni CA, Boparai N, McCullough AJ. Nonalcoholic Fatty Liver Disease in Patients With Type 2 Diabetes. Clin Gastroenterol Hepatol 2004;2:262-265. doi: 10.1016/S1542-3565(04)00 014-X.

[11] Adams LA, Sanderson S, Lindor KD, Angulo P. The histological course of nonalcoholic fatty liver disease: a longitudinal study of 103 patients with sequential liver biopsies. J Hepatol 2005;42:132-138. doi: 10.1016/j.jhep .2004.09.012.

[12] Adams LA, Lymp JF, St Sauver J, Sanderson SO, Lindor KD, Feldstein A, et al. The natural history of nonalcoholic fatty liver disease: A population-based cohort study. Gastroenterology 2005;129:113-121. doi: 10.1053/j.gastro .2005.04.014.

[13] de Ledinghen V, Ratziu V, Causse X, Le Bail B, Capron D, Renou C, et al. Diagnostic and predictive factors of significant liver fibrosis and minimal lesions in patients with persistent unexplained elevated transaminases. A prospective multicenter study. J Hepatol 2006;45:592-599. doi: 10.1016 /j.jhep.2006.05.008.

[14] Angulo P, Keach JC, Batts KP, Lindor KD. Independent predictors of liver fibrosis in patients with nonalcoholic steatohepatitis. Hepatology 1999;30: 1356-1362. doi: 10.1002/hep.510300604.

[15] Raynard B, Balian A, Fallik D, Capron F, Bedossa P, Chaput JC, et al. Risk factors of fibrosis in alcohol-induced liver disease. Hepatology 2002;35:635638. doi: 10.1053/jhep.2002.31782.

[16] Hessheimer AJ, Forner A, Varela M, Bruix J. Metabolic risk factors are a major comorbidity in patients with cirrhosis independent of the presence of hepatocellular carcinoma. Eur J Gastroenterol Hepatol 2010;22:12391244. doi: 10.1097/MEG.0b013e32833aa19b.

[17] Chen CL, Yang HI, Yang WS, Liu CJ, Chen PJ, You SL, et al. Metabolic factors and risk of hepatocellular carcinoma by chronic hepatitis B/C infection: A follow-up study in Taiwan. Gastroenterology 2008;135:111-121. doi: 10.1053/j.gastro.2008.03.073.

[18] Lai MS, Hsieh MS, Chiu YH, Chen TH. Type 2 diabetes and hepatocellular carcinoma: A cohort study in high prevalence area of hepatitis virus infection. Hepatology 2006;43:1295-1302. doi: 10.1002/hep.21208.

[19] Adami HO, Ekbom A, Chow WH, Nyren O, Wolk A, Berne C, et al. Excess risk of primary liver cancer in patients with diabetes mellitus - Response. J Natl Cancer Inst 1997;89:327-328. doi: 10.1093/jnci/89.4.327.

[20] Wideroff L, Gridley G, Mellemkjaer L, Chow WH, Linet M, Keehn S, et al. Cancer incidence in a population-based cohort of patients hospitalized with diabetes mellitus in Denmark. J Natl Cancer Inst 1997;89:1360-1365. doi: 10.1093/jnci/89.18.1360.

[21] Hassan MM, Hwang LY, Hatten CJ, Swaim M, Li D, Abbruzzese JL, et al. Risk factors for hepatocellular carcinoma: Synergism of alcohol with viral hepatitis and diabetes mellitus. Hepatology 2002;36:1206-1213. doi: 10.1053/jhep.2002.36780.

[22] Davila JA, Morgan RO, Shaib Y, McGlynn KA, El-Serag HB. Diabetes increases the risk of hepatocellular carcinoma in the United States: a population based case control study. Gut 2005;54:533-539. doi: 10.1136/gut.2004.052167.

[23] Hassan MM, Curley SA, Li DH, Kaseb A, Davila M, Abdalla EK, et al. Association of Diabetes Duration and Diabetes Treatment With the Risk of Hepatocellular Carcinoma. Cancer 2010;116:1938-1946. doi: 10.1002 /cncr.24982.

[24] Kleiner DE, Brunt EM, Van Natta M, Behling C, Contos MJ, Cummings OW, et al. Design and validation of a histological scoring system for nonalcoholic fatty liver disease. Hepatology 2005;41:1313-1321. doi: 10.1002/hep .20701.

[25] Association Diabetes Association. Diagnosis and classification of diabetes mellitus. Diabetes Care 2014;37(Suppl 1):S81-S90. doi: $10.2337 / \mathrm{dc14}$ S081.

[26] Alberti KG, Zimmet $P$, Shaw J. Metabolic syndrome - a new world-wide definition. A Consensus Statement from the International Diabetes Federation. Diabetic Medicine 2006;23:469-480. doi: 10.1111/j.14645491.2006.01858.x.

[27] Stone NJ, Bilek S, Rosenbaum S. Recent National Cholesterol Education Program Adult Treatment Panel III update: adjustments and options. Am J Cardiol 2005;96:53E-59E. doi: 10.1016/j.amjcard.2005.06.006.

[28] Aronow WS. Updated National Cholesterol Education Program III guidelines. J Am Med Dir Assoc 2005;6:160-161. doi: 10.1016/j.jamda.2004.12.022.
[29] Bruix J, Sherman M, American Association for the Study of Liver Diseases. Management of hepatocellular carcinoma: an update. Hepatology 2011;53: 1020-1022. doi: 10.1002/hep.24199.

[30] D'Hoore W, Sicotte C, Tilquin C. Risk adjustment in outcome assessment: the Charlson comorbidity index. Methods Inf Med 1993;32:382-387.

[31] Charlson ME, Pompei P, Ales KL, MacKenzie CR. A new method of classifying prognostic comorbidity in longitudinal studies: development and validation. J Chronic Dis 1987;40:373-383. doi: 10.1016/0021-9681(87)90171-8.

[32] Brunt EM, Janney CG, Di Bisceglie AM, Neuschwander-Tetri BA, Bacon BR. Nonalcoholic steatohepatitis: a proposal for grading and staging the histological lesions. Am J Gastroenterol 1999;94:2467-2474. doi: 10.1111/ j.1572-0241.1999.01377.x.

[33] Intraobserver and interobserver variations in liver biopsy interpretation in patients with chronic hepatitis C. The French METAVIR Cooperative Study Group. Hepatology 1994;20:15-20. doi: 10.1002/hep.1840200104.

[34] Wong R, Cheung R, Ahmed A. Nonalcoholic steatohepatitis is the most rapidly growing indication for liver transplantation in patients with hepatocellular carcinoma in the U.S. Hepatology 2014;59:2188-2195. doi: 10.1002 /hep.26986.

[35] Nakahara $T$, Hyogo $H$, Yoneda $M$, Sumida $Y$, Eguchi $Y$, Fujii $H$, et al. Type 2 diabetes mellitus is associated with the fibrosis severity in patients with nonalcoholic fatty liver disease in a large retrospective cohort of Japanese patients. J Gastroenterol 2013;49:1477-1484. doi: 10.1007/s00535-0130911-1.

[36] Doycheva I, Patel N, Peterson M, Loomba R. Prognostic implication of liver histology in patients with nonalcoholic fatty liver disease in diabetes. J Diabetes Complications 2013;27:293-300. doi: 10.1016/j.jdiacomp.2012 .10 .008 .

[37] Ryan M, Wilson A, Slavin J, Best J, Jenkins A, Desmond P. Associations Between Liver Histology and Severity of the Metabolic Syndrome in Subjects With Nonalcoholic Fatty Liver Disease. Diabetes Care 2005;28:1222-1224. doi: $10.2337 /$ diacare.28.5.1222

[38] Fujino Y, Mizoue T, Tokui N, Yoshimura T. Prospective study of diabetes mellitus and liver cancer in Japan. Diabetes-Metabolism Research and Reviews 2001;17:374-379. doi: 10.1002/dmrr.214

[39] Balbi M, Donadon V, Ghersetti M, Grazioli S, Valentina GD, Gardenal R, et al. Alcohol and HCV chronic infection are risk cofactors of type 2 diabetes mellitus for hepatocellular carcinoma in Italy. Int J Environ Res Public Health 2010;7:1366-1378. doi: 10.3390/ijerph7041366.

[40] Donadon V, Balbi M, Casarin P, Vario A, Alberti A. Association between hepatocellular carcinoma and type 2 diabetes mellitus in Italy: Potential role of insulin. World J Gastroenterol 2008;14:5695-5700. doi: 10.3748/ wjg.14.5695.

[41] Roden M. Mechanisms of Disease: hepatic steatosis in type 2 diabetes pathogenesis and clinical relevance. Nat Clin Pract Endocrinol Metab 2006;2: 335-348. doi: 10.1038/ncpendmet0190.

[42] Crespo J, Cayon A, Fernandez-Gil P, Hernandez-Guerra M, Mayorga M, Dominguez-Diez A, et al. Gene expression of tumor necrosis factor alpha and TNF-receptors, p55 and p75, in nonalcoholic steatohepatitis patients. Hepatology 2001;34:1158-1163. doi: 10.1053/jhep.2001.29628.

[43] Jonsson JR, Moschen AR, Hickman IJ, Richardson MM, Kaser S, Clouston AD, et al. Adiponectin and its receptors in patients with chronic hepatitis C. ] Hepatol 2005;43:929-936. doi: 10.1016/j.jhep.2005.05.030.

[44] Svegliati-Baroni G, Ridolfi F, Di Sario A, Casini A, Marucci L, Gaggiotti G, et al. Insulin and insulin-like growth factor-1 stimulate proliferation and type I collagen accumulation by human hepatic stellate cells: Differential effects on signal transduction pathways. Hepatology 1999;29:1743-1751. doi: 10.1002/hep.510290632.

[45] Marra F, Bertolani C. Adipokines in Liver Diseases. Hepatology 2009;50:957969. doi: 10.1002/hep.23046

[46] Saito T, Chiba T, Yuki K, Zen Y, Oshima M, Koide S, et al. Metformin, a diabetes drug, eliminates tumor-initiating hepatocellular carcinoma cells. PLoS One 2013;8:e70010. doi: 10.1371/journal.pone.0070010.

[47] Facciorusso A. The influence of diabetes in the pathogenesis and the clinical course of hepatocellular carcinoma: recent findings and new perspectives. Curr Diabetes Rev 2013;9:382-386. doi: 10.2174/1573399811309 9990068.

[48] Butt Z, Jadoon NA, Salaria ON, Mushtaq K, Riaz IB, Shahzad A, et al. Diabetes mellitus and decompensated cirrhosis: risk of hepatic encephalopathy in different age groups. J Diabetes 2013;5:449-455. doi: 10.1111/17530407.12067.

[49] Quintana JO, García-Compean D, González JA, Pérez JZ, González FJ, Espinosa LE, et al. The impact of diabetes mellitus in mortality of patients with compensated liver cirrhosis-a prospective study. Ann Hepatol 2011;10 56-62. 